\title{
FrA TRADIZIONE E INNOVAZIONE: LA “Storia dei Filosofi” in Età Antica, Medievale E Moderna
}

\author{
Gregorio Piaial
}

\begin{abstract}
RIASSUNTO: Il graduale passaggio dall'antica "storia dei filosofi" alla moderna "storia della filosofia" viene qui ricostruito nelle sue fasi essenziali, alla luce della dialettica fra tradizione e innovazione, che caratterizza il dialogo filosofico inteso in senso diacronico. Ma in che senso è ancora possibile, oggi, parlare di una "storia della filosofia" distinta sia dall'attività filosofico-ermeneutica sia dalla ricerca strettamente storica, qual è quella condotta dalla Intellectual history?
\end{abstract}

PAROLE-CHIAVI: Tradizione. Innovazione. Storiografia filosofica.

\section{INTRODUZIONE}

Si può dire che la dimensione dialogica, ovvero la disponibilità ad ascoltare le ragioni dell'altro e di avviare con lui un confronto razionalmente fondato, sia costitutiva del "far filosofia", al di là delle diverse accezioni che lo stesso concetto di "filosofia" ha assunti nel corso dei secoli. Le forme del dialogo in campo filosofico si possono ridurre essenzialmente a due, a seconda che tale dialogo si svolga in senso sincronico o diacronico. Nel primo caso il dialogo consiste in un dibattito fra contemporanei, che espongono le proprie tesi e cercano di argomentarle mediante un confronto diretto (gli interlocutori sono seduti allo stesso tavolo o nella stessa aula, fisicamente vicini, oppure sono collegati in videoconferenza) o indiretto, mediante interventi scritti (con "risposte", repliche, controrepliche, postille ecc.) pubblicati sulle riviste filosofiche e di varia umanità. Ma il dialogo filosofico

\footnotetext{
${ }^{1}$ Nato a Belluno nel 1944, Gregorio Piaia è professore ordinario di Storia della filosofia presso l'Università di Padova ed è socio dell'Istituto Veneto di Scienze Lettere ed Arti, dell'Accademia Galileiana di Scienze Lettere ed Arti in Padova e dell'Ateneo di Treviso. La sua attività di ricerca si è svolta essenzialmente lungo tre direttrici: a) il pensiero politico ed etico-religioso fra tardo medioevo e Rinascimento (Marsilio da Padova, Nicolò da Cusa, Erasmo da Rotterdam, Tommaso Moro...); b) la teoria e la storia della storiografia filosofica; c) la cultura filosofica nel Veneto fra Sette ed Ottocento. Ha inoltre collaborato alla nuova edizione della Enciclopedia filosofica (Milano 2006, voll. 12), dirigendo la sezione sui secoli XVII e XVIII.
} 
avviene anche con gli autori del passato, sia recente sia lontano, che non sono più fisicamente in vita ma che continuano a vivere sul piano intellettuale grazie alle loro opere, dalle quali continuiamo ad attingere tutta una serie di informazioni, suggestioni, stimoli, ipotesi, provocazioni: insomma, una vastissima gamma di testimonianze sul modo (anzi sui modi) con cui in passato si è "fatto filosofia", da cui non si può prescindere se non si vuole cadere nell'ingenuità o nella presunzione, che non sarebbero atteggiamenti propriamente filosofici.

Questa seconda maniera di dialogare è ovviamente più complessa, dando luogo ad articoli e a saggi più o meno corposi e a volumi monografici spesso ponderosi. La complessità (o meglio la difficoltà) nasce dal fatto che l'autore di cui esaminiamo gli scritti non è più in grado di rispondere alle nostre domande, che possono riferirsi alla sua biografia intellettuale e alla genesi e composizione delle sue opere, ma anche - e soprattutto - al senso esatto dei termini e dei concetti che egli usa, al significato "vero" delle sue dottrine e dei suoi stessi silenzi, ossia al "detto" e al "non detto": che cosa ha "veramente" pensato e detto il filosofo $\mathrm{X}$ ? perché lo ha detto nella forma che ci è pervenuta? e che rapporto c'è fra il testo lasciatoci da un filosofo e la "persona" stessa del filosofo, che non esiste più ma che ci parla ancora attraverso i suoi scritti? e che cosa potrebbe ancora dirci il filosofo X, vissuto ieri o l'altro ieri, rispetto ai problemi filosofici che oggi ci stanno a cuore?... Alle dottrine filosofiche del passato (paragonabili alle res gestae, per usare un'espressione in uso nelle discipline storiche) ci si accosta dunque con un'operazione assai complessa e articolata (la historia rerum gestarum, ossia, nella fattispecie, la storiografia filosofica) che inizia con l'individuazione e la definizione sul piano filologico dei documenti filosofici che ci sono pervenuti (ossia "tràditi", dal latino tradere, da cui il concetto di tradizione) e prosegue con un delicato lavoro di ricostruzione, contestualizzazione, comprensione, ma anche di analisi critica, di valutazione e di interpretazione, che può condurre a un modo diverso $\mathrm{e}$ nuovo di "leggere" un'opera filosofica e di inserirla nel dibattito contemporaneo a chi compie tale lettura (è il concetto di innovazione).

Fra tradizione e innovazione si è venuto instaurando un rapporto che è ad un tempo complementare e dialettico. Senza l'approccio filologico, che garantisce la permanenza o la sopravvivenza stessa della tradizione, lo studio delle filosofie del passato cadrebbe nel puro arbitrio, ma tale approccio non è esaustivo: per comprendere il pensiero di un autore è necessario anche un lavoro di ri-costruzione storico-filosofica, che è sempre aperto ad interpretazioni innovative, le quali dipendono dalla capacità di penetrazione e dalla sensibilità del singolo studioso, oltre che dal mutato clima intellettuale. Se poi ci si accosta ad un autore del passato con l'obiettivo primario di trovarvi uno spunto o un supporto alle proprie tesi filosofiche, la "forbice" rispetto al momento filologico 
si divarica ancor più, sino a fare della storia della filosofia un grande deposito di concetti e di idee che - Heidegger insegna - si possono interpretare con il massimo di "originalità" (o di unilateralità?) sino a ridurre il dialogo con l'altro ad un monologo individuale e ad un palese sfruttamento delle posizioni altrui. Una forte istanza innovativa può infine indurre a respingere in blocco - almeno a parole - il rapporto stesso con le filosofie del passato, per puntare decisamente a una "nuova filosofia", che si vuole profondamente diversa da quella tradizionale. Ecco allora i due poli fra i quali si consuma il nostro rapporto con il passato filosofico: ascoltare e rivivere la tradizione, oppure innovare radicalmente rispetto ad essa. Sono emblematiche, in proposito, le posizioni incarnate da due autori, Machiavelli e Descartes, assai diversi fra loro e che pure siamo soliti porre all'inizio del pensiero moderno. La prima è racchiusa nella lettera che Nicolò Machiavelli, confinato in un piccolo villaggio di contadini toscani, scrisse all'amico Francesco Vettori in data 10 dicembre 1513; in essa l'ideale del dialogo con gli antichi autori, maestri di "humanità", viene espresso in maniera quanto mai efficace e suggestiva:

Venuta la sera, mi ritrovo in casa, et entro nel mio scrittoio; et in sull'uscio mi spoglio quella veste cotidiana, piena di fango et di loto, e mi metto panni reali et curiali; et rivestito condecentemente entro nelle antique corti degli antiqui huomini, dove, da loro ricevuto amorevolmente, mi pasco di quel cibo, che solum è mio, et ch'io nacqui per lui; dove io non mi vergogno parlare con loro, e domandarli della ragione delle loro actioni, et quelli per loro humanità mi rispondono. (MACHIAVELLI, 1961, p. 305).

Altrettanto celebri e significative sono alcune considerazioni che Descartes fece nella prima parte del Discours de la méthode. Dopo aver riconosciuto - ecco l'eredità umanistica, che ci riporta a Machiavelli - che "[...] la lettura di libri buoni è come una conversazione con i loro autori, i quali furono tra le persone più illustri (les plus honnêtes gens) del passato, ed è anzi una conversazione meditata (étudiée), nella quale essi ci scoprono il meglio dei loro pensieri”, Descartes non esita a rilevare i limiti dell'approccio al passato, ovvero alla tradizione:

Conversare con gli uomini di altri secoli è quasi lo stesso che viaggiare: certo, è bene saper qualcosa dei costumi dei vari popoli per giudicare meglio dei nostri, e non stimare ridicolo e irragionevole (contre raison) tutto ciò ch'è contrario alle nostre abitudini, come credono coloro che non hanno visto mai nulla; ma quando s'impiega troppo tempo a viaggiare, si diventa alla fine stranieri nel proprio paese (étranger en son pays), e così chi è troppo curioso delle cose del passato diventa, per lo più, molto ignorante di quelle presenti. (DESCARTES, 1962, p. 6; 1991, p. 47). 
In che misura questa polarità fra tradizione e innovazione, così bene espressa da Machiavelli e Descartes, si è esercitata sul modo di ricostruire il passato della filosofia? Detto in altri termini: se la tradizione, in quanto incentrata sulla fedele trasmissione di un corpus dottrinale, è essenzialmente legata all'attività filologica o più genericamente erudita, quali furono gli aspetti innovativi che un approccio di natura più teorica introdusse di volta in volta nella "storia della filosofia" in quanto historia rerum gestarum, sino a farne in età moderna una disciplina autonoma, dotata di un proprio statuto epistemologico? E quale può essere oggi la collocazione della "storia della filosofia", alla luce del percorso compiuto in particolare negli ultimi quattro secoli?

\section{NON SOlO ERUdizIONE: DOSSOGRAFIA E "VITE EI FILOSOFI" NELL'ETÀ ANTICA E MEDIEVALE}

L'esigenza di fare riferimento alle tesi dei predecessori e quindi di ordinarle e classificarle ai fini di una discussione critica appare già chiaramente in Platone: ad es. nel Sofista (242c-243c, 245e-246d) quando lo straniero di Elea, trattando dell'essere e del non-essere, menziona le posizioni di chi (Ferecide di Siro?) ammette tre elementi oppure due (Archelao) o uno solo (gli Eleati) oppure una unione di uno e di molteplice insieme (Eraclito ed Empedocle), per poi accennare alla "battaglia di giganti" intorno alla nozione di "essere", che vede contrapposti i "figli della terra" (i materialisti) agli "amici delle forme" (242c-243c, 245e-246d); oppure nel Protagora (316d-e, 342a-343b), là dove il protagonista fa una rassegna degli antichi predecessori dell'arte sofistica, o dove Socrate replica ironicamente che la filosofia più antica si trova a Creta ed a Sparta, e cita i Sette Sapienti.

È tuttavia in Aristotele che quest'operazione retrospettiva acquista ampiezza e sistematicità, al punto che si può vedere in lui non solo l'iniziatore dell'antica storiografia filosofica, ma anche il creatore di un modello di approccio alle filosofie del passato, un vero e proprio archetipo, significativamente ripreso - fra i tanti dallo stesso Hegel. È noto che Aristotele era solito accompagnare le sue trattazioni con una rassegna delle "opinioni" (doxai) sostenute dai pensatori precedenti: all'inizio della Fisica e del De anima (ma anche nel De caelo e nel De generatione et corruptione) e soprattutto nel libro I della Metafisica lo Stagirita ricostruisce le posizioni dei suoi predecessori secondo il metodo "dialettico", inteso non in senso hegeliano ma platonico-aristotelico, per cui la

[...] contrapposizione, nonché lo sviluppo delle conseguenze di ciascuna delle due opinioni opposte, cioè l'aporia e il successivo diaporèsai, sono per lui certamente indispensabili per giungere alla verità, cioè all'euporia, ma non 
coincidono necessariamente con le posizioni storicamente assunte dai filosofi precedenti. (BERTI, I986, p. 104).

In Aristotele la ricostruzione storica ha dunque una chiara finalità speculativa: di volta in volta essa concorre a individuare i tre principi (materia, forma, privazione), a definire compiutamente l'anima, a dimostrare la finitezza ed eternità dell'universo, oppure - è il caso del libro I della Metafisica - serve da verifica sul piano storico di una dottrina, qual è quella delle quattro cause, già elaborata in precedenza sul piano strettamente teorico (cf. Metaph., 983a-b). Al tempo stesso, però, la ricchezza d'informazioni e il carattere organico e unitario fecero di queste trattazioni una fonte privilegiata per lo studio dei presocratici e delle origini stesse della filosofia, anche se taluni studiosi hanno denunciato le deformazioni e "falsificazioni" operate da Aristotele e che si sarebbero poi surrettiziamente introdotte nella storiografia filosofica sino ai nostri giorni.

L'influsso degli schemi aristotelici fu determinante nel costituire la tradizione dossografica, che ebbe inizio con il successore di Aristotele alla guida del Peripato, Teofrasto di Ereso, autore dell'opera in 18 libri Physikon doxai (Le dottrine fisiche), ove le "opinioni" dei filosofi sono così raccolte e ordinate: gli dèi, il mondo, i fenomeni meteorologici, la fisiologia, la psicologia. Di tale raccolta sono rimasti solo frammenti, in particolare un'ampia sezione De sensibus (DIELS, $1965^{4}$, p. 102-144) Da Teofrasto ha inizio una ricca produzione dossografica, in gran parte perduta, che attraverso i Vetusta placita e l'opera di Aezio giunge ai Placita philosophorum dello ps. Plutarco e alle consimili opere dello ps. Galeno e di Giovanni Stobeo, influendo anche sugli autori latini (Cicerone, Varrone) e cristiani (Ippolito, Ermia). Alla scuola di Aristotele risale anche l'origine di un altro genere storiografico, e cioè le biografie dei filosofi, benché già l'Apologia di Socrate si presenti ricca non solo di notizie sulla vita, ma anche di indicazioni sull'indole e sulla condotta di Socrate, che corrispondono appieno al concetto di bios, inteso essenzialmente come "[...] l'individuale maniera di vivere rispecchiante la natura interiore” (UNTERSTEINER, 1980, p. 223). Nel Peripato l'interesse per le ricerche di storia sia naturale sia letteraria si fonde con la ricerca dei "tipi", già abbozzata da Aristotele nell'Etica Nicomachea e sviluppata da Teofrasto nei Caratteri.

Accanto al genere dossografico e biografico, nel mondo antico si sviluppò anche il genere diadochistico, imperniato sulla successione (diadokè) degli scolarchi all'interno delle diverse scuole filosofiche. L'iniziatore di questa "storiografia delle scuole" fu Sozione di Alessandria (III-II sec. a.C.), cui si deve la distinzione - rimasta in auge fino al sec. XIX - fra due grandi scuole del pensiero greco: la Ionica, derivata da Talete (Mileto, com'è noto, faceva parte delle colonie ioniche dell'Asia minore) e l'Italica, derivata da Pitagora, ch'era nativo dell'isola di Samo nell'arcipelago delle 
Cicladi, ma s'era stabilito a Crotone nella Magna Grecia. Negli autori vissuti in età imperiale i tre generi sopra indicati tendono a fondersi insieme, come nella Raccolta delle vite e delle dottrine dei filosofi di Diogene Laerzio (sec. III d.C.), l'unica opera storiografica dell'antichità giunta quasi completa sino a noi. Divisa in dieci libri (i primi sette sulla filosofia ionica, i rimanenti tre su quella italica), l'opera è preceduta da un proemio in cui fra l'altro si rivendica l'origine greca (e non "barbara") della filosofia, ed è costruita su uno schema ricorrente: di ogni filosofo Diogene Laerzio segnala la stirpe, il luogo di nascita, il carattere, le massime e i detti, la cronologia, il modo in cui ha lasciato questa vita, le invenzioni, gli aneddoti, le lettere, una sintesi delle dottrine, il testamento (qualora ci sia) e infine gli eventuali omonimi (DIOGENE LAERZIO, 1991, p. XVIII-XIX).

Fra gli autori cristiani dei primi secoli l'interesse per la conoscenza e l'esame critico degli antichi filosofi fu assai vivo in s. Giustino, Clemente d'Alessandria, Origene, Eusebio di Cesarea e s. Agostino. Sullo sfondo di tale interesse v'era il problema dei rapporti fra il messaggio giudaico-cristiano e la cultura pagana, bollata da Tertulliano quale matrice di eresie. È significativo, al riguardo, l'atteggiamento espresso da s. Agostino in più luoghi della sua opera e particolarmente nei primi dieci capitoli del libro VIII del De civitate Dei, ove lo svolgimento della filosofia antica dalle origini a Platone viene ripercorso sulla scorta delle Tusculanae e degli Academica di Cicerone e di manuali tardo-antichi di storia della filosofia. Ne risulta una sorta di "dossografia speculativa", in cui la rassegna di opinioni intorno alla natura di Dio porta ad esaltare la superiorità di Platone rispetto a tutti i filosofi pagani, per la sua vicinanza alla prospettiva cristiana. Frutto della storiografia ellenistico-romana e della più matura riflessione patristica, la trattazione di Agostino avrebbe esercitato un influsso profondo e duraturo: se lungo tutto il medioevo latino, data la scarsità o l'assenza delle fonti antiche, essa finì con l'assurgere al rango di fonte autorevole sul pensiero greco, assai rilevante fu pure la sua incidenza su alcuni autori del Cinque-Seicento, che dalla concezione agostiniana della storia e della "sapienza" trassero ragioni ideali e categorie interpretative per l'indagine storico-filosofica.

Per quanto priva dello status di disciplina autonoma, nel medioevo una storiografia filosofica è tuttavia esistita. Una prima versione delle Vite laerziane, il cui testo non ci è pervenuto, risulta già compiuta da Enrico Aristippo fra il 1156 e il 1160 . Nell'universo culturale dell'età di mezzo circolano nuclei cospicui di materiali storico-filosofici, inseriti entro generi letterari differenti (storie universali, enciclopedie come lo Speculum di Vincenzo di Beauvais, summae dottrinali, poemi didascalici come l'Entheticus de dogmate philosophorum di Giovanni di Salisbury, raccolte biografiche...) e utilizzati con finalità differenti, ma omogenei e ben ordinati quanto alla loro struttura interna. In particolare gli 
excursus storici contenuti nella Summa philosophiae dello ps. Grossatesta e nell'Opus maius di Ruggero Bacone segnano un netto e significativo distacco dagli schemi della storiografia antica, in quanto la ricostruzione del passato filosofico non si limita al pensiero greco (con quella specie di "introduzione" che era rappresentata dalla filosofia "barbara"), ma si prolunga sino all'età contemporanea, superando il contrasto tra la philosophia come prodotto esclusivo del mondo pagano e il messaggio cristiano fondato sulla Scrittura. La produzione storico-filosofica medievale ha dunque una sua fisionomia, che non è riducibile a una modesta sopravvivenza di talune forme della storiografia antica, quali potrebbero apparire a prima vista il Compendiloquium de vitis illustrium philosophorum di Giovanni di Galles (II metà del sec. XIII) e il Liber de vita et moribus philosophorum poetarumque veterum, tradizionalmente attribuito a Walter Burley e risalente ai primi decenni del Trecento (PIAIA, 2006, cap. 4).

\section{Dalla historia philosophica come genere aUtonomo all'identità di FILOSOFIA E STORIA DELLA FILOSOFIA}

La riscoperta e valorizzazione in età umanistico-rinascimentale di tutte le antiche scuole filosofiche, unitamente alla nuova traduzione latina delle Vite di Diogene Laerzio ad opera di Ambrogio Traversari, risalente agli anni 1427-1433, diedero luogo a una ricca produzione storiografica, che riprese e sistematizzò i modelli ereditati dal mondo antico. Pervaso dall'idea platonicocristiana di philosophia perennis e dalla prospettiva agostiniana, che trasmette una forte consapevolezza del "donde si viene" e del "verso dove si procede", questo riacquistato patrimonio di notizie e di schemi si tradurrà in un nuovo genere letterario: la storia "generale" della filosofia, dalle origini sino all'età contemporanea, che si sviluppa in particolare nell'area protestante (assai sensibile, ad un tempo, all'erudizione storica e ai temi di teologia della storia) ed ha il primo grande esempio negli Historiae philosophicae libri septem del tedesco Georg Horn, pubblicati a Leida nel 1655, ma già redatti intorno al 1640. In quello stesso anno veniva stampato a Londra il primo dei 4 voll. della History of philosophy dell'inglese Thomas Stanley (opera molto vasta, ma limitata alla filosofia antica); nel 1657 58 apparivano postumi all'Aja i De philosophia et philosophorum sectis libri duo di Johannes Gerhard Vossius, mentre un vero e proprio repertorio bibliografico, accompagnato da riflessioni metodologiche, veniva offerto da Johannes Jonsius nei De scriptoribus historiae philosophicae libri quattuor (Francoforte, 1659), segno dell'ormai raggiunta autonomia e maturità di questo genere letterario. Questa letteratura storico-filosofica è dovuta a filologi classici, ad eruditi, a studiosi di storia universale e non a filosofi in senso stretto, tant'è vero che la historia 
philosophica è vista come un settore della più ampia "polistoria", che ha per oggetto tutti i prodotti letterari.

La nascita della "nuova filosofia" è in un primo tempo estranea, se non ostile, all'interesse per le filosofie del passato: Descartes fonda la possibilità di un filosofare rigoroso a prezzo della storia, dichiarando di voler azzerare il rapporto con il passato, e Malebranche manifesta una totale indifferenza per l'indagine storica: "Ė inutile sapere ciò che Aristotele ha creduto sull'immortalità dell'anima, benché sia molto utile sapere che l'anima è immortale" (MALEBRANCHE, 1945-1946, I, p. 154). Questa divaricazione fra verità e storia, e quindi tra la filosofia e il suo passato, viene però a comporsi tra la fine del Seicento e i primi decenni del Settecento, quando l'istanza critica ereditata da Cartesio e lo spirito sistematico di Leibniz (aperto tuttavia agli "antichi” e non solo ai "moderni") trasformano la tradizionale storia delle vite e delle sètte dei filosofi in una storia della filosofia, che prende avvio da una base storica e filologica ma è incentrata sulla "connessione" logica delle dottrine e sul "giudizio" intorno alla loro validità, e si ispira a un rinnovato eclettismo contrapposto allo spirito di sètta. La historia philosophica - operazione essenzialmente erudita, ove il termine philosophica si riferisce all'oggetto d'indagine - si trasforma così in una storia "critica" (o "filosofica" nel senso del metodo) della filosofia, grazie anche all'apporto del celebre Dictionnaire historique et critique (1697) di Pierre Bayle, che presenta molte voci su pensatori antichi e moderni. I lavori storiografici del Buddeus, le riflessioni metodologiche della Einleitung zur historia philosophica che introduce gli "Acta philosophorum" (Halle, 1715-1727) di Christoph A. Heumann, la Histoire critique de la philosophie (Amsterdam, 1737) di André-François BoureauDeslandes e soprattutto la monumentale Historia critica philosophiae (5 voll., Leipzig, 1742-44; II ed., in 6 voll., 1766-67) di Joh. Jacob Brucker segnano i momenti più significativi di questa evoluzione, che garantisce alla storiografia filosofica una collocazione autonoma e un preciso statuto epistemologico (PIAIASANTINELLO, 2011, capp. 2-3, 5-6, 8).

Con il Brucker, che godette di vasta fortuna in tutta Europa, si apre la serie delle grandi storie della filosofia dei secc. XVIII e XIX, che accompagnano l'eccezionale sviluppo assunto dalla speculazione filosofica in Germania. Se i 6 voll. del Geist der spekulativen Philosophie (Marburg, 1791-97) di Dieterich Tiedemann si muovono sul terreno della storia "pragmatica" (aperta alle prospettive della francese histoire de l'esprit humain, antenata di quella che sarebbe poi stata la Kulturgeschichte) e sono caratterizzati da una chiara idea di progresso e da un richiamo diretto ai testi, la "svolta kantiana" inflù profondamente sulla storiografia filosofica, alimentando anche un vivace dibattito teorico sulla "filosofia della storia della filosofia”, che rese obsoleti l'eclettismo del Brucker e l'apparato erudito che 
questi aveva ereditato dagli storiografi secenteschi. Kant non compose opere specifiche di storia della filosofia, ma il breve abbozzo di una "Storia della ragion pura” con cui si chiude la prima Critica lascia intendere com' egli considerasse "[...] incompiuto il sistema della filosofia trascendentale in mancanza di una esplicita trattazione sistematica - condotta da un punto di vista non empirico - del lavoro compiuto dalla ragione nel corso della storia” (MICHELI, I988, p. 880). Tale istanza trovò una risposta adeguata nelle opere di due kantiani: il Lehrbuch der Geschichte der Philosophie (9 vol., Göttingen, 1796-1804) e la Geschichte der neuern Philosophie (6 voll., ivi 1800-1805) di Joh. Gottlieb Buhle, la Geschichte der Philosophie (11 voll., Leipzig, 1798-1819) e il Grundriss der Geschichte der Philosophie (ivi 1812) di Wilhelm Gottlieb Tennemann. Questi due autori in particolare il Tennemann - muovono dalla convinzione che il criticismo kantiano segni un cambiamento decisivo nella storia del pensiero, consentendo la fondazione della filosofia come scienza, e procedono ad una ricostruzione a priori del corso storico della filosofia, imperniata su alcuni possibili "sistemi tipo" che corrispondono alle diverse facoltà e disposizioni dell'uomo (le strutture trascendentali) e che si ripetono con poche varianti nel corso della storia, secondo un andamento più ciclico che progressivo, almeno fino alla "rivoluzione" operata da Kant. Si tratta dunque di una storia costruita prevalentemente per linee interne, secondo una tendenza che caratterizzerà anche la successiva storiografia idealistica.

Le Lezioni sulla storia della filosofia di Hegel apparvero postume a Berlino fra il 1833 e il 1836, tratte dai quaderni di appunti stesi dai suoi allievi. Mentre i kantiani, sulla base di una rilettura del trascendentale in chiave psicologistica, avevano inteso la storia della filosofia quale sviluppo dell'autocoscienza umana, per Hegel è la ragione universale o Idea al centro di tale sviluppo. Di qui l'identificazione di filosofia e di storia della filosofia, concepita - quest'ultima - come l'automanifestarsi della ragione in quanto "Spirito del Mondo", una "ragione" che è l'assoluto stesso. In questa rinnovata prospettiva metafisicoteologica la coincidenza fra la deduzione logica delle categorie del pensiero assoluto e la successione storica dei sistemi di filosofia è garantita dal movimento dialettico, capace di far comprendere il "concreto" storico e di conferire unità, senso e direzione allo sviluppo (Entwicklung) della storia. Risulta così sciolta la "contraddizione interiore" fra verità e storia, rilevata in una celebre pagina della Einleitung alle Lezioni:

Giacché la filosofia si propone di intendere ciò che è immutabile, eterno, in sé e per sé: il suo scopo è la verità. La storia invece racconta ciò che fu in un determinato tempo e poi sparì in un altro tempo, perché fu sostituito da qualcosa d'altro. Noi partiamo infatti dal presupposto che la verità sia eterna: essa non rientra nell'àmbito di ciò che passa, quindi non ha alcuna storia. $\mathrm{E}$ 
se tuttavia essa ha una storia, [...] in questa storia non può trovarsi la verità, poiché la verità non è cosa transitoria. (HEGEL, 1982, p. 43).

Nella prospettiva hegeliana l'attività storico-filosofica appare dunque sublimata e trasfigurata, ma anche dissolta, privata di quell'identità disciplinare ch'essa aveva acquistato dopo la sua rinascita moderna e alla quale avevano contribuito sia l'erudizione secentesca sia il progetto settecentesco di una storia "critica". E così, proprio quando l'istanza "filosofica" nel fare storia della filosofia - da molti propugnata a partire da Leibniz - trova in Hegel la sua più completa ed esaltante attuazione, la storiografia filosofica rischia di smarrire la propria specificità e consistenza.

\section{QUale FUTURo PER LA STORIA DELLA FILOSOFIa?}

L'impronta radicalmente innovativa impressa da Hegel alla sua ricostruzione del passato filosofico si presta a molteplici osservazioni. In primo luogo questo tipo di approccio non rappresenta una novità assoluta, come già s'è notato accennando alla "lettura teoretica" cui Aristotele aveva a suo tempo sottoposto i pensatori a lui precedenti (basti pensare a Talete, al quale viene applicata una nozione - quella di causa "materiale", distinta da quella formale - che era estranea al pensatore di Mileto.) Il fatto poi che Hegel rinfacciasse agli storici della filosofia a lui anteriori di essere stati ben poco filosofi non significa che questi storiografi fossero soltanto degli eruditi o dei filologi, bensì che la loro prospettiva filosofica, che pure era ben presente ed operante, non coincideva con quella di Hegel: anche l'eruditissimo Brucker aveva un suo orientamento speculativo, che Hegel, bontà sua, riteneva però del tutto inconsistente e quindi non-filosofico... D'altro canto il sistematico ricorso al principio dialettico fa sì che in Hegel risulti superata la stessa opposizione fra tradizione e innovazione, dal momento che il passato filosofico non viene emarginato o confinato nella "galleria degli errori", ma viene ricompreso - sia pure con qualche sforzo e con qualche taglio - entro una superiore linea di sviluppo che pretende di spiegare e far comprendere quel complesso movimento delle idee che nel processo di autocoscienza dell'Idea trova la sua giustificazione ultima. Ma cosa resterebbe allora del ricco e variegato patrimonio di dottrine che la tradizione ci ha trasmesso grazie all'opera degli eruditi e dei filologi, e che vanno colte nel loro contesto storico e culturale anziché schiacciate da uno schema affascinante ma fuorviante? .

Dopo Hegel - sintetizzo in maniera brutale - gli storici della filosofia reagirono al dogma della corrispondenza fra concetti logici e successione storica e rivendicarono l'autonomia del lavoro storico-filosofico, pur mantenendo parecchi elementi dell'impianto hegeliano, a cominciare dall'idea (che entrerà in crisi nel 
corso del secondo Novecento) di uno sviluppo dialettico ed unitario della storia del pensiero. Non occorre certo sottolineare il ruolo svolto nel contempo da Dilthey e dallo storicismo tedesco nello sviluppo di una linea di ricerca ispirata alla Kulturgeschichte. Dal canto suo la History of ideas, affacciatasi quasi in sordina, si sviluppa nell'ultimo Novecento come Intellectual history (RANDALL, 1963; SCHNEIDER, 2005), sino a fare quasi tutt'uno con quei settori della storiografia filosofica che non si sentono strettamente funzionali alla riflessione teoretica e che prendono anzi le distanze da essa. Per converso il recente, grande sviluppo della "filosofia ermeneutica" ha segnato la forte ripresa di un approccio speculativo ai pensatori del passato, che riduce al minimo la conquistata distinzione tra il "far filosofia" e il "fare storia della filosofia". In breve, comunanza di oggetto (le dottrine filosofiche del passato, ossia la "tradizione"), ma diversità di approccio, di metodo e di finalità... Si potrebbe allora ipotizzare che al termine della sua parabola la disciplina "storia della filosofia" sia destinata a smarrire la sua autonomia, riaccostandosi al grande alveo materno della filosofia tout court oppure assumendo la veste e i metodi della intellectual history. Fine, dunque, della historia philosophica?

È tempo di prendere posizione. Pur sentendomi assai prossimo, per forma mentis e per itinerario di ricerca, alla intellectual history, non credo tuttavia che essa possa escludere in toto il momento filosofico. Fra i prodotti dell'umano ingegno, come si diceva nel nostro Settecento, esiste sempre una zona di contatto e di scambio. Anche la più rigorosa indagine storica è accompagnata da quella terenziana curiositas ("homo sum, humani nihil a me alienum puto") che non a caso venne ripresa da Montaigne e che non si esaurisce certo nel gusto per l'aneddotica o nel puro divertissement intellettuale. Essa comporta pur sempre un com-partecipare, un com-patire, un con-sentire che sono l'altra faccia del distacco con cui dobbiamo guardare ai "reperti" filosofici del passato. Ed è un compartecipare che nasce da quello che, a mio avviso, dovrebbe essere l'atteggiamento di fondo (ovvero la "filosofia") dello storico della filosofia: un mix di eclettismo e di moderato scetticismo, ove l'onesta consapevolezza dell'impossibilità di raggiungere $l a$ verità e di poterla quindi esibire come la mia verità non si traduce in un atteggiamento negativo di rifiuto o di insofferenza, bensì in un habitus di apertura alle più svariate espressioni dell'umano pensare. Era questa, a prescindere dagli autori qui citati, la lezione offerta a cavallo del 1700 da un singolare historienphilosophe quale fu Pierre Bayle, cui non a caso si richiamò Eugenio Garin in una pagina che a quarant'anni di distanza merita ancor oggi d'essere riletta e meditata:

In realtà è proprio quest'abito di tolleranza intellettuale, unito all'idea di una pluralità di punti di vista possibili, che alimenta una storia che vuole conoscere con estrema fedeltà le varie posizioni. Se la verità è, in quanto raggiungimento definitivo, le désespoir così dello storico come del filosofo; se la conoscenza è una ricerca più o meno plausibile, ma non mai conclusa, si giustifica non solo 
l'indugio curioso su tutte le ricerche, ma l'ansia puntigliosa di indagarle tutte sino in fondo, di coglierne fin dove è possibile il senso, di scandagliarle nei particolari e di saggiarne la consistenza criticandole. GARIN, 1970, p. 266).

È una prospettiva che richiede una buona dose di esprit de finesse rispetto allo spirito geometrico o metafisico o parateologico che hanno spesso contrassegnato le moderne ricostruzioni storico-filosofiche; ed è in questa prospettiva che il dialogo con la tradizione filosofica può oggi mantenersi vivo e produttivo, al di là delle illusioni o delle presunzioni intellettuali che gli storici della filosofia hanno spesso accarezzato in questi ultimi tre secoli.

PIAIA, Gregorio. Between tradition and innovation: the "History of the Philosophers" in Ancient, Medieval and Modern Eras. Trans/Form/Ação, Marília, v. 34, n.3, p. 3-15, 2011.

ABSTRACT: In this essay, the gradual transition from ancient "history of the philosophers" to modern "history of philosophy" is presented according to its essential steps and in the light of the dialectic between tradition and innovation that characterizes any philosophical dialogue considered in a diachronic sense. At the same time, however, the essay raises the question of the sense according to which it is nowadays still possible to think of a "history of philosophy" as a research activity distinct both from philosophical hermeneutics and from strictly historical research, such as the one carried out by intellectual history.

KEYWORDS: Tradition. Innovation. Historiography of Philosophy.

\section{Bibliografia}

BERTI, E. Sul carattere "dialettico" della storiografia filosofica di Aristotele. In: Storiografia e dossografia nella filosofia antica, a cura di G. Cambiano. Torino: Tirrenia, 1986, p. 101-125.

DESCARTES, R. Discours de la méthode, texte et commentaire par É. Gilson. Paris: Vrin, $1962^{3}$.

. Discorso sul metodo, a cura di A. Carlini. Roma-Bari: Laterza, $1991^{28}$.

DIELS, H. Doxographi Graeci, Berlin: W. de Gruyter, 1879, $1965^{4}$.

DIOGENE LAERZIO, Vite dei filosofi, a cura di M. Gigante. Milano: TEA, 1991.

GARIN, E. La storia "critica” della filosofia nel Settecento. In: . Dal Rinascimento all'Illuminismo. Studi e ricerche. Pisa: Nistri Lischi, 1970 [Le Lettere, Firenze 1993²].

HEGEL, G.W.F. Introduzione alla storia della filosofia, a cura di A. Plebe. Roma-Bari: Laterza, 1982. 
MACHIAVELLI, N. Lettere, a cura di F. Gaeta. Milano: Feltrinelli, 1961 (Opere, 6).

MALEBRANCHE. De la recherche de la vérité, éd. G. Lewis. Paris: Vrin, 1945-46 [1974²].

MICHELI, G. La svolta kantiana. In: Storia delle storie generali della filosofia, a cura di G. Santinello, Vol. 3: Il secondo Illuminismo e l'età kantiana. Padova: Antenore, I988, pp. 879-957.

PIAIA, G. Entre história e imáginario. O passado da filosofia na Idade Média, tr. M. N. Costa e L.A. De Boni. Porto Alegre: Edipucrs, 2006.

; SANTINello, G. (Ed.). Models of the History of Philosophy, Vol. 2: From Cartesian Age to Brucker. Dordrecht: Springer, 2011.

RANDALL, J. H. Arthur O. Lovejoy and the History of Ideas. Philosophy and Phenomenological Research, Vol. 23, p. 475-479, 1963.

SCHNEIDER, U. J. Intellectual History in a Global Age. Journal of the History of Ideas, vol 66, p. 155-167, 2005.

UNTERSTEINER, M. Problemi di filologia filosofica, a cura di L. Sichirollo e M. Venturi Ferriolo. Milano: Cisalpino-Goliardica, 1980.

recebido em: 17.06.2011

aprovado em: 14.08.2011 